\title{
Sozial interagierende Roboter in der Pflege
}

\author{
Kathrin Janowski, Hannes Ritschel, Birgit Lugrin und Elisabeth André
}

\begin{abstract}
Zusammenfassung
In naher Zukunft werden Roboter immer häufiger in den Lebensbereichen von Alltagsnutzerinnen und -nutzern anzutreffen sein. Sehr deutlich wird dies am Beispiel von Haushalts- und Pflegerobotern, denen im Hinblick auf eine alternde Gesellschaft verstärkt die Rolle des Mitbewohners und Weggefährten zukommen wird. Damit einher geht der Wunsch, die Mensch-Roboter-Kommunikation menschzentrierter zu gestalten. Es geht nicht mehr lediglich um die effiziente Durchführung von Aufgaben, sondern auch darum, für den Menschen ein angenehmes Interaktionserlebnis zu schaffen und ein Vertrauensverhältnis zu dem Roboter herzustellen. Der nachfolgende Artikel diskutiert den aktuellen Stand in Forschung und Entwicklung im Bereich sozial interagierender Roboter, die über multimodale soziale Hinweisreize mit pflegebedürftigen Menschen kommunizieren.
\end{abstract}

K. Janowski $(\bowtie) \cdot H$. Ritschel $\cdot$ E. André

Universität Augsburg, Augsburg, Deutschland

E-Mail: kathrin.janowski@informatik.uni-augsburg.de

H. Ritschel

E-Mail: hannes.ritschel@informatik.uni-augsburg.de

E. André

E-Mail: andre@hcm-lab.de

B. Lugrin

Universität Würzburg, Würzburg, Deutschland

E-Mail: birgit.lugrin@uni-wuerzburg.de

(C) Der/die Autor(en) 2018

O. Bendel (Hrsg.), Pflegeroboter, https://doi.org/10.1007/978-3-658-22698-5_4 


\subsection{Einleitung}

Soziale Roboter, die den Menschen bei unterschiedlichen Aufgaben unterstützen oder einfach nur zur Unterhaltung dienen, dringen immer mehr in die Alltagsbereiche von Nutzerinnen und Nutzern vor. Diese Entwicklung zeichnet sich aufgrund des demografischen Wandels vor allem im Bereich der Pflege ab. Vor allem in Japan werden Pflegeroboter bereits sehr erfolgreich in der Altenpflege eingesetzt. Aber auch in Europa findet dieses Thema zunehmend Beachtung. Bei älteren Menschen ist die Akzeptanz geringer als bei jüngeren Menschen. Dennoch zeigen Umfragen, dass immer mehr Seniorinnen und Senioren dem Einsatz von Pflegerobotern positiv gegenüberstehen. Beispielsweise konnten sich bei einer repräsentativen Forsa-Umfrage, welche im April 2016 im Auftrag des BMBF durchgeführt wurde, $83 \%$ der Befragten grundsätzlich vorstellen, im Alter einen Serviceroboter in ihrem eigenen Zuhause zu nutzen, um dort länger wohnen zu können (Forsa. Politik- und Sozialforschung GmbH 2016).

In der Pflege können Roboter in den unterschiedlichsten Bereichen zum Einsatz kommen (siehe Abb. 4.1). Dazu gehören körperliche Aufgaben wie das Aufräumen und Putzen der Wohnung oder Hilfe bei persönlicher Hygiene (Srinivasa et al. 2010; King et al. 2010), aber auch kognitive Aufgaben wie die Erinnerung an Medikamente oder soziale Aufgaben wie das Erzählen von Geschichten (Huijnen et al. 2011; Mizak et al. 2017; Sidner et al. 2013). Die vom Roboter erbrachte Unterstützung kann älteren Menschen unter anderem ermöglichen, länger zu Hause ein selbstbestimmtes Leben zu führen.

Vielfach wird argumentiert, dass maschinelle Interaktionspartner auf erhebliche Akzeptanzprobleme bei Alltagsnutzern stoßen werden, wenn sie sich nicht an die zwischenmenschliche Kommunikation annähern. Dies gilt vor allem in Bereichen, in denen technische Systeme dem Nutzer als Gefährte, Berater oder Lehrer zur Seite stehen sollen. In den letzten Jahren wurden erhebliche Anstrengungen unternommen, um die Ausdrucksmöglichkeiten von maschinellen Interaktionspartnern zu verbessern.

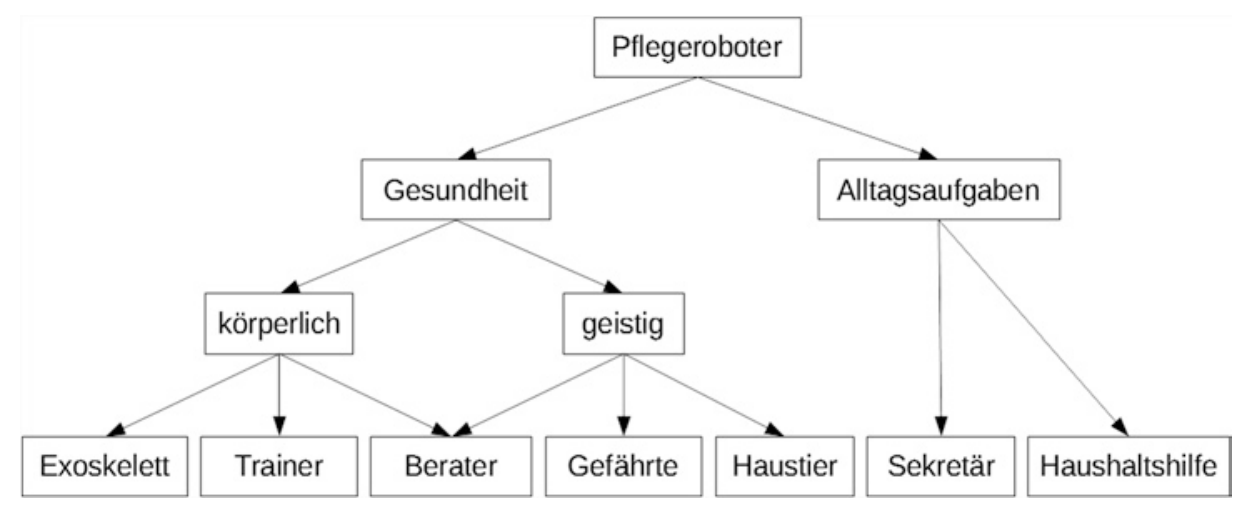

Abb. 4.1 Ein Überblick über verschiedene Arten von Assistenzrobotern 
Eine besondere Herausforderung besteht in der fein abgestimmten Koordination der multimodalen Verhaltensweisen eines Roboters wie Körperhaltung, Gestik und Sprache, um soziale Interaktionen zwischen Mensch und Roboter zu ermöglichen.

Im vorliegenden Beitrag wird ein Überblick zu sozial interagierenden Robotern in der Pflege gegeben. Sozial interagierende Roboter unterscheiden sich von reinen Servicerobotern dadurch, dass sie während der Ausführung von Diensten Verhaltensweisen emulieren, die an der zwischenmenschlichen Kommunikation orientiert sind. So würde eine sozial interagierende Hebehilfe eine Person nicht nur umlagern, sondern beispielsweise auch mitfühlend nachfragen, ob die Person nun bequem liegt. Bemerkt sei, dass die Interaktion nicht unbedingt in natürlicher Sprache erfolgen muss. In einigen Fällen kann es durchaus sinnvoll sein, ausschließlich über soziale und emotionale Hinweise mit einem Artefakt zu interagieren. Ein bekanntes Beispiel hierfür ist die mit einem weichen Fell ausgestattete Kuschelrobbe Paro, die mittels Sensorik auf Berührungen und Geräusche von Nutzern reagiert.

Mit der Entwicklung sozial interaktiver Roboter geht der Wunsch einher, die Mensch-Maschine-Kommunikation anthropozentrischer und persönlicher zu gestalten. Das mühsame Erlernen von Kommandos entfällt, da die Menschen so mit der Maschine interagieren können, wie sie es aus der zwischenmenschlichen Interaktion gewöhnt sind. Es geht jedoch nicht nur um einfache Bedienung. Die Nutzung von technischen Artefakten soll zu einer positiven Erfahrung werden. Im Idealfall kommt es zu einer vertrauensvollen Beziehung zwischen Mensch und Roboter. D. h., der Roboter wird nicht nur als Werkzeug gesehen, sondern seine Rolle wandelt sich zum persönlichen Begleiter oder sogar zum Freund.

Der vorliegende Beitrag skizziert den aktuellen Forschungs- und Entwicklungsstand zu sozial interagierenden Robotern. Abschn. 4.2 identifiziert Anforderungen, welche für sozial interagierende Roboter erfüllt sein müssen, um bei Nutzerinnen und Nutzern auch längerfristig auf Akzeptanz zu stoßen. Dabei liegt der Fokus auf der Sicht der Pflegebedürftigen. In Abschn. 4.3 werden grundlegende Techniken zur Realisierung wichtiger Eigenschaften sozial interagierender Roboter wie Empathie und sozial situiertes Lernen erläutert. Besondere Aufmerksamkeit gilt dabei den sprachlichen Fähigkeiten von Robotern, durch welche eine intuitive Kommunikation zwischen Mensch und Maschine ermöglicht werden soll. Abschn. 4.4 illustriert Einsatzmöglichkeiten sozial interagierender Roboter in der Rolle von Physiotherapeuten, Gesundheitsberatern, Spielgefährten und Kuscheltieren. In Abschn. 4.5 geht es um die Gestaltung von sozial interagierenden Robotern, wobei wir nicht nur ihr Erscheinungsbild, sondern vor allem auch ihre kommunikativen Verhaltensweisen betrachten. Abschn. 4.6 gibt einen Überblick zu Studien zur Wirkung von sozial interagierenden Robotern. Das Kapitel schließt mit einer Konklusion. 


\subsection{Anforderungen und Herausforderungen}

Der Pflegekontext bringt einige besondere Anforderungen an soziale Roboter mit sich. Die Interaktion mit einem sozialen Roboter sollte auf die Interaktion mit älteren Menschen abgestimmt sein und das mühsame Erarbeiten von maschinennahen Interaktionsweisen ersparen. Ob sich zwischen Mensch und Roboter eine Beziehung entwickeln und diese über einen längeren Zeitraum aufrechterhalten werden kann, hängt nicht nur von der Art der physikalischen Verkörperung und der Mechanik des Roboters ab. Wichtiger ist ein glaubwürdiges Verhalten. D. h., der Roboter muss als eigenständige Persönlichkeit mit konsistenten Verhaltensweisen wahrgenommen werden. So sollte beispielsweise seine Stimme zu seiner Erscheinung passen. Im Kontext der Pflege ist ein sensibles Verhalten des Roboters von zentraler Bedeutung. Insbesondere muss er in der Lage sein, auf die Bedürfnisse und Empfindungen älterer Menschen einzugehen. Längerfristig soll durch häufige Interaktionen ein Vertrauensverhältnis zwischen Mensch und Roboter aufgebaut werden. Damit dies gelingt, sollte der Roboter jederzeit zur Verfügung stehen und ein Gedächtnis besitzen, um sich an vorangegangene Interaktionen oder Ereignisse erinnern zu können. Schließlich sollte er aus der Interaktion mit Nutzerinnen und Nutzern lernen und ein individuell an ältere Menschen und ihre Präferenzen und Einschränkungen angepasstes soziales Verhalten zeigen.

\subsubsection{Glaubwürdiges Verhalten}

Roboter sind physikalische Objekte mit Form und Gestalt. Viele Merkmale, die auf verschiedene menschliche Sinne einwirken, definieren das Erscheinungsbild der Maschine, so etwa die Größe des Roboters, Art oder Geschwindigkeit von Bewegungen, das Sound-Design und Klang der Stimme oder allgemein das Design des Gehäuses mit verschiedenen Materialien und Formen. Obwohl neue Technologien, zum Beispiel Sprachassistenten wie Amazon Alexa, auch soziale Assistenzsysteme rein auf Basis von Sprache ohne menschenähnliche Gestalt ermöglichen, trägt die Verkörperung, ähnlich wie bei virtuellen Charakteren, doch wesentlich zum Gesamteindruck des Systems bei. Menschen tendieren dazu, Maschinen mit lebensähnlicher Gestalt oder Bewegung Intentionen, Ziele, Emotionen und Persönlichkeiten zuzuschreiben (Reeves und Nass 1998). Deshalb muss auch bei sozialen Pflegerobotern berücksichtigt werden, wie glaubhaft ein Roboter seine Intentionen und Ziele kommuniziert. Darüber hinaus sollte ein Roboter als eigenständige Persönlichkeit mit einem emotionalen Verhalten wahrgenommen werden. In den letzten Jahren wurden enorme Anstrengungen unternommen, die Ausdrucksfähigkeit von Robotern zu erhöhen. Die Erfahrung hat jedoch gezeigt, dass eine realistische Erscheinung in Verbindung mit einer eindrucksvollen Mechanik nicht ausreicht, um eine dauerhafte Bindung zum Nutzer aufzubauen. Maschinelle Interaktionspartner mit anthropomorphen Fähigkeiten profitieren zwar zunächst vom Neuheitseffekt, verlieren 
aber nach kurzer Zeit ihren Reiz für den Nutzer. Wichtiger für die Akzeptanz von Robotern ist deren Fähigkeit, mit Menschen auf sozial angemessene Art und Weise zu interagieren.

Ein langfristiges Ziel ist das Herstellen einer Beziehung zwischen Mensch und Roboter. Dies wird begünstigt durch eine attraktive Roboterpersönlichkeit, die die Interaktion interessanter gestaltet und erstrebenswerter macht (Breazeal 2004). Persönlichkeit umfasst Charakter, Verhalten, Temperament, Emotionen sowie mentale Eigenschaften des Menschen. Um diese Merkmale zu kategorisieren, hat sich in der Psychologie die „Big-Five“-Taxonomie (McCrae und Costa 2008) bzw. das „Fünf-Faktoren-Modell“ etabliert, wobei Persönlichkeit durch fünf Dimensionen beschrieben wird: Offenheit, Gewissenhaftigkeit, Extraversion, Verträglichkeit und Neurotizismus.

Für den Ausdruck von Persönlichkeit stehen der Maschine ähnliche Möglichkeiten zur Verfügung wie dem Menschen. Zum einen spiegelt sich Persönlichkeit wesentlich in der Sprache wider, sodass mithilfe natürlichsprachlicher Generierung Formulierungen entsprechend der Ausprägung der fünf Dimensionen gebildet werden können (Mairesse und Walker 2011), zum anderen stehen auch - je nach Verkörperung des Roboters Mimik und Gestik zur Verfügung. Auch die Kombination von beidem - verbalem und nonverbalem Verhalten - wurde bereits für virtuelle Charaktere untersucht (Walker et al. 2014). Der Ausdruck von Persönlichkeit auf Basis von natürlichsprachlicher Generierung für einen sozialen Roboter kommt beispielsweise bei Ritschel et al. (2017) zum Einsatz.

\subsubsection{Sozial- und emotionssensitive Roboter}

Die Bereitschaft und Fähigkeit, sich in die Einstellungen und Emotionen anderer Menschen einzufühlen, ist nicht nur in der zwischenmenschlichen Kommunikation wichtig, sondern sollte auch bei der Entwicklung sozialer Roboter berücksichtigt werden. Zwar können Maschinen keine echte Empathie fühlen, dennoch sollten sie dazu in der Lage sein, ein entsprechendes Verhalten nachzubilden. Zu dieser Aufgabe gehört zunächst das Wahrnehmen des menschlichen emotionalen Zustands, etwa auf Basis sozialer Signale, die beispielsweise mithilfe von Video- oder Audiodaten erfasst werden können. Anschließend sollte eine adäquate Reaktion gezeigt werden, in der der Roboter selbst Zeichen von Empathie zum Ausdruck bringt. Neben dem gezielten Einsatz von Emotionen gehört auch das Zeigen von Verständnis dazu. D. h., der Roboter muss Situationen aus der Perspektive eines Nutzers bewerten, um dessen Emotionen zu verstehen.

Um eine Verbindung zum Menschen herstellen und aufrechterhalten zu können, sollten soziale Roboter dazu in der Lage sein, Interesse am Fortgang einer Interaktion zu zeigen und zu demonstrieren, dass sie dem Verlauf der Kommunikation folgen. Im Idealfall sollten Roboter ihre kommunikativen Verhaltensweisen an denen des Menschen ausrichten, wobei der gemeinsame Redehintergrund stets aufs Neue bestätigt und abgesichert wird. Dies erfordert den gezielten Einsatz verbaler und nichtverbaler sozialer Signale wie etwa das Herstellen von Blickkontakt, Nicken oder 
Kopfschütteln (Mehlmann et al. 2014). Umgekehrt sollte der Roboter auch in der Lage sein zu erkennen, wann das Engagement des Nutzers nachlässt, und Gegenmaßnahmen ergreifen. Eine große Herausforderung besteht darin, dass der Nutzer fehlendes Engagement nicht immer explizit zum Ausdruck bringt, sondern implizit durch soziale Hinweisreize wie Abwenden des Blicks oder fehlende Rückkopplungssignale kommuniziert.

\subsubsection{Längerfristige Interaktionen}

Sidner et al. (2013) haben den Begriff eines „Always-On“-Roboters geprägt. Dies bedeutet, dass ein Roboter dem Nutzer permanent zur Verfügung steht und ihn wahrnimmt, ohne dass dieser den Roboter jedes Mal einschalten muss. Dazu muss der Roboter in der Lage sein, zu verstehen, wann der Nutzer interagieren möchte und wann nicht. Eine Voraussetzung hierfür ist die Fähigkeit, die menschliche Anwesenheit wahrnehmen und darauf reagieren zu können. Der Roboter sollte fähig sein, aus Eigeninitiative auf sich aufmerksam machen zu können, um bei Bedarf eine Interaktion zu veranlassen.

Roboter in der Rolle von Gefährten sollten sich gesprächsübergreifend an frühere Inhalte erinnern, um darauf Bezug zu nehmen oder Nutzerinnen und Nutzer bei Bedarf mit Informationen versorgen zu können. Dabei ist aber nicht nur das Erinnern selbst von Bedeutung, sondern auch das gezielte Vergessen von Inhalten früherer Gespräche oder Ereignisse. Ein menschlicher Gefährte wird sich in den seltensten Fällen an den genauen Wortlaut von Gesprächen erinnern können. Bei einem künstlichen Gefährten wäre dies kein Problem, aber nicht unbedingt wünschenswert. Da Vergessen menschlich ist, verliert ein Roboter, der zu jedem Zeitpunkt alle Geschehnisse inklusive aller Details rekapitulieren kann, unter Umständen an Glaubhaftigkeit. Darüber hinaus besteht die Gefahr, dass ein solcher Roboter Unbehagen hervorruft, da Menschen sich in ihrer Privatsphäre verletzt fühlen. Nach Lim et al. (2009) erlaubt das Vergessen einem Roboter auch, sich - ähnlich wie der Mensch - auf wichtige Aspekte zu fokussieren, was langfristig ein konsistentes Verhalten und den Ausdruck von Persönlichkeit begünstigen kann.

Der Aufbau einer vertrauensvollen Beziehung zwischen Nutzer und Agent ist eine wesentliche Voraussetzung für eine längerfristige Nutzung. Bickmore und Cassell setzen auf Small Talk (Bickmore und Cassell 1999), um eine gemeinsame Grundlage zwischen Menschen und Agenten aufzubauen und den gegenseitigen Zusammenhalt zu stärken.

\subsubsection{Sozial situiertes Lernen}

Um den individuellen Vorlieben, Präferenzen, aber auch Einschränkungen des menschlichen Gegenübers entgegenzukommen, sollte ein sozialer Roboter in der Lage sein, diese wahrzunehmen und zu erlernen, sich entsprechend der jeweiligen Situation passend zu verhalten. Hierbei müssen nach Tapus et al. (2007) Kurzzeit- und Langzeitänderungen unterschieden werden. Zum einen müssen die aktuellen Bedürfnisse des Gegenübers 
berücksichtigt werden, zum anderen soll die Interaktion auch nachhaltig ansprechend gestaltet werden, damit der Nutzer auch nach Monaten oder Jahren noch engagiert bleibt. Darüber hinaus ist zu berücksichtigen, dass sich die Interessen, Bedürfnisse und Fähigkeiten von Nutzern mit der Zeit verändern. Um eine an den jeweiligen Menschen angepasste Interaktion bereitstellen zu können, muss der Roboter in der Lage sein, vom Nutzer zu lernen und seine eigenen Fähigkeiten an die Persönlichkeit, Stimmung und Präferenzen des Menschen anzupassen. Breazeal (2004) hat für dieses Verhalten den Begriff ,,sozial situiertes Lernen“ geprägt. In Abschn. 4.3.3 wird auf aktuelle Verfahren eingegangen, die sozial situiertes Lernen ermöglichen.

\subsection{Technische Voraussetzungen}

Die Umsetzung von bedienfreundlichen Nutzerschnittstellen für Senioren und Seniorinnen ist mit enormen technischen Herausforderungen verbunden. Da diese Generation nicht an den Umgang mit technischen Systemen gewöhnt ist, ist mit Berührungsängsten zu rechnen. Des Weiteren müssen körperliche Einschränkungen wie verminderte Sehfähigkeit oder zittrige Hände bedacht werden. Daher ist eine natürliche und intuitive Nutzerschnittstelle wünschenswert, welche beispielsweise über natürliche Sprache oder Haptik funktioniert.

Sprachbasierte Steuerung bietet den Vorteil, dass Menschen mit dieser Form der Kommunikation vertraut sind. Durch jüngste Fortschritte auf dem Gebiet der Spracherkennung ist es möglich geworden, solche Schnittstellen im Alltag einzusetzen. Beispielsweise testete das Front Porch Center for Innovation and Wellbeing über sechs Monate hinweg den Einsatz von Amazon Alexa in einer Seniorengemeinde, mit überwiegend positiven Rückmeldungen (Mizak et al. 2017).

Reis et al. (2017) verglichen die Sprachassistenten Amazon Alexa, Google Assistant, Microsoft Cortana und Apple Siri im Hinblick auf den Einsatz im Haushalt von Senioren. Dabei lag der Schwerpunkt auf den angebotenen Diensten, zu welchen diese jeweils die zentrale Schnittstelle bilden. Zum Repertoire der Agenten gehören praktische Aufgaben wie Terminplanung, Wettervorhersage oder die Suche nach Nachrichten und Informationen, Unterhaltungsfunktionen wie Musikwiedergabe oder Spiele, aber vor allem auch die Kommunikation mit Freunden und Familie. Dies deckt sich mit den Funktionen, welche von der Zielgruppe gewünscht werden (Mizak et al. 2017; Pino et al. 2015).

Durch den Einzug in den häuslichen Bereich und die ständige Präsenz im Alltag ihrer Nutzer wird sich auch die Rolle dieser Technologie vom reinen Werkzeug hin zum persönlichen Begleiter oder sogar Freund wandeln. Damit werden emotionale und soziale Faktoren bei der Mensch-Technik-Interaktion immer wichtiger. Das Bedürfnis danach zeigt sich auch darin, dass Senioren und Seniorinnen neben der vorgeschriebenen Interaktion auch dazu tendieren, sich mit Robotern über Persönliches zu unterhalten, wie beispielsweise Unzufriedenheit mit der Lebenssituation oder den Angehörigen (Sabelli et al. 2011). 


\subsubsection{Sprachliche Fähigkeiten}

Die erste Hürde bei der natürlichsprachlichen Kommunikation ist, ein klares Audiosignal von einer einzelnen Person zu erhalten. Dazu werden meistens mehrere Mikrofone gleichzeitig genutzt, um den Sprecher zuerst im Raum zu lokalisieren. Anschließend ist es möglich, die Mikrofone optimal auf diese Person auszurichten (zum Beispiel durch Drehung des Roboterkopfes) oder Störgeräusche aus anderen Richtungen rechnerisch auszufiltern. Der Roboter Reeti ${ }^{1}$ hat beispielsweise zwei Mikrofone unterhalb der Augenkameras, während bei $\mathrm{NaO}^{2}$ und $\mathrm{Pepper}^{3}$ jeweils vier über die Oberseite des Kopfes verteilt sind. Geräte aus Amazons Echo-Reihe ${ }^{4}$, der Schnittstelle zu Alexa, verwenden je nach Modell vier, sieben oder sogar acht Mikrofone.

Menschliche Sprache ist umfangreich und komplex, sodass es kaum möglich ist, den gesamten Wortschatz und alle grammatikalisch möglichen Formulierungen in einem Rechenmodell abzudecken. Umgangssprache oder regionale Dialekte erschweren dies zusätzlich, und manche Nutzer halten sich schlichtweg nicht an sprachliche Gepflogenheiten, sei es aus Bequemlichkeit oder mangelnder Kenntnis. In der Praxis ist der Dialog mit solchen Systemen daher oft noch auf einfach strukturierte Fragen und konkrete Befehle begrenzt. Aktuelle Sprachassistenten nutzen die Rechenzentren der zugehörigen Firmen, um derartige Funktionen anzubieten (Chung et al. 2017). Durch Zugriff auf deren umfangreiche Datensätze und hohe Rechenleistung ist es möglich, aufwendigere maschinelle Lernverfahren zur Interpretation natürlicher Sprache einzusetzen, für welche kleineren Geräten wie Mobiltelefonen oder dem Amazon Echo die nötige Hardware fehlt. Auch Roboter wie Nao und Pepper verwenden diesen Ansatz zur Spracherkennung. ${ }^{5}$ Ein Nachteil dieser Lösung ist allerdings die Abhängigkeit von einer stabilen Internetverbindung, was besonders in ländlichen Gegenden oft schwierig ist. Dazu kommen massive Bedenken bezüglich Privatsphäre und Datenschutz, da derartige Geräte theoretisch jederzeit Tonaufnahmen an die Herstellerfirma senden können. Falls die Verbindung nicht ausreichend gesichert ist, besteht außerdem die Gefahr von unautorisierten Zugriffen oder Manipulation durch Außenstehende (Chung et al. 2017). Diese Nachteile können durch die Verwendung quelloffener Software oder privat eingerichteter Server umgangen werden, was allerdings mit deutlich höherem Entwicklungsaufwand verbunden ist.

Damit ein sozialer Agent dem Nutzer in natürlicher Sprache antworten kann, muss zuerst ein Text dafür erzeugt werden. Dazu kann beispielsweise ein vorgegebenes Textgerüst mit aktuellen Informationen vervollständigt werden, was sich besonders für funktionale

\footnotetext{
${ }^{1}$ http://reeti.fr/index.php/en/detailen.

${ }^{2} \mathrm{http}: / /$ doc.aldebaran.com/2-1/family/robots/microphone_robot.html.

${ }^{3}$ http://doc.aldebaran.com/2-5/family/pepper_technical/microphone_pep.html.

${ }^{4}$ https://www.amazon.de/dp/B06ZXQV6P8.

${ }^{5}$ https://developer.softbankrobotics.com/us-en/documents/top-100-questions.
} 
Aufgaben wie Wetterberichte eignet. Für soziale Dialoge werden dagegen häufig Datensätze mit menschlichen Äußerungen erstellt, aus denen das System lernen soll, welcher Satz in der jeweiligen Situation angebracht ist (Serban et al. 2018). Anschließend wird der Text mittels einer künstlich erzeugten Stimme ausgegeben. Diese basiert auf Audiodaten, welche zuvor von einem menschlichen Sprecher aufgezeichnet wurden. Zurzeit gibt es zwei verbreitete Ansätze (Aylett et al. 2017) dafür: Der sogenannte Unit-Selection-Ansatz zerlegt diese Aufnahmen in elementare Bausteine wie typische Redewendungen, einzelne Worte oder Phoneme und setzt sie neu zusammen. Bei parametrischer Synthese wird mit den Audiodaten ein statistisches Modell trainiert, welches zwar weniger natürliche Ergebnisse liefert, aber dafür weniger darauf angewiesen ist, dass die benötigten Lautkombinationen oft genug in den Sprachaufnahmen vorkamen. Üblicherweise können auch die Lautstärke und Geschwindigkeit der Sprachsynthese an die Bedürfnisse des Nutzers angepasst werden, damit diese so verständlich wie möglich ist. Um verschiedenen Einsatzzwecken gerecht zu werden, bieten manche Firmen außerdem nicht nur Stimmen unterschiedlichen Geschlechts an, sondern auch Kinderstimmen, verschiedene Akzente oder Varianten mit emotionaler Färbung. Allerdings konzentriert sich das Angebot dafür bisher auf Englisch und Sprachen mit ähnlicher weltweiter Verbreitung.

Bemerkt sei, dass sozial interagierende Roboter nicht nur Dialoge mit dem Menschen führen, um eine funktionale Aufgabe zu erfüllen, wie z. B. das Erinnern an das Einnehmen von Medikamenten, sondern auch, um eine vertrauensvolle Beziehung zu dem Menschen herzustellen. Bickmore und Cassell haben unterschiedliche Techniken zum Aufbau eines Vertrauensverhältnisses (Bickmore und Cassell 2001) untersucht, wie beispielsweise Small Talk (Bickmore und Cassell 1999). Das von Bickmore und Cassell implementierte System berücksichtigt bei der Planung eines Gesprächs die zwischenmenschliche Nähe, das jeweilige Thema sowie dessen Relevanz, Aufgabenziele und logische Vorbedingungen. Ziel bei der Auswahl des nächsten Gesprächsabschnitts ist die Maximierung des Nutzervertrauens. Im Rahmen einer durchgeführten Studie konnte gezeigt werden, dass Small Talk bei extrovertierten Personen einen positiven Einfluss auf deren Vertrauen in das System hatte.

\subsubsection{Empathisches Verhalten}

Die Entwicklung von Maschinen mit der Fähigkeit zur Empathie stellt Forschung und Entwicklung vor erhebliche Herausforderungen. Zum einen werden ausgefeilte Techniken zur Analyse von Gefühlszuständen eines Nutzers benötigt, die sich meist unbewusst in dessen Gesichtsausdruck, Gestik, Körperhaltung und Sprache widerspiegeln. Zum anderen muss der maschinelle Interaktionspartner über die Fähigkeit verfügen, sich in die Gefühle eines Nutzers, wie z. B. Stress oder Ärger, hineinzuversetzen und angemessen darauf zu reagieren. In diesem Abschnitt soll auf die technischen Voraussetzungen zur Realisierung empathischer Roboter eingegangen werden. Für eine ausführlichere Darstellung verweisen wir auf (André 2014). 
Um auf den Gefühlszustand des Nutzers reagieren zu können, muss ein künstlicher Assistent diesen zuallererst erkennen können. Menschen drücken ihre Gefühle auf vielfältige Weise aus, beispielsweise durch Mimik, Tonfall oder Körperhaltung.

Aktuelle Ansätze zur Interpretation von Gesichtsausdrücken zerlegen diese meistens in einzelne Bausteine, welche jeweils ein markantes Merkmal im Gesicht verformen, wie beispielsweise die Mundwinkel oder die Augenbrauen. Ein verbreitetes Schema dafür ist das von Ekman und Friesen entwickelte „Facial Action Coding System“ (Ekman et al. 2002), welches sich an der Gesichtsmuskulatur orientiert und jeder charakteristischen Bewegung eine so genannte „Action Unit“ zuordnet. Mithilfe von Bildverarbeitungsalgorithmen können diese Verformungen auf zwei- oder dreidimensionalen Kamerabildern identifiziert werden.

Akustische Emotionserkennung betrachtet verschiedene Merkmale wie beispielsweise die Energie und das Frequenzspektrum einer Spracheingabe (Vogt et al. 2008). Mittels maschineller Lernverfahren können bei geschauspielerten Emotionen unter Laborbedingungen sehr gute Ergebnisse erzielt werden. Natürlich auftretende Emotionen sind dagegen subtiler und schwieriger zu erkennen (Vogt und André 2005). Realistischere Trainingsdaten erhält man, indem man Gefühle durch externe Reize provoziert, zum Beispiel durch emotionale Videos oder manipulierte Spielsituationen.

Für eine zielgerichtete Reaktion muss das System außerdem in der Lage sein, Rückschlüsse über die Ursache der beobachteten Gefühlslage zu ziehen. Beispielsweise ist dies wichtig für die Unterscheidung, ob der Agent den Nutzer nur aufmuntern soll oder selbst einen Fehler gemacht hat und sich entschuldigen muss. Dazu muss er relevante Ziele des Nutzers kennen, Ereignisse in Bezug auf diese Ziele bewerten und wenn möglich auch den Verursacher der Ereignisse identifizieren. Streng genommen erfolgt die Bewertung bei einem empathischen Agenten nicht aus seiner eigenen Perspektive, sondern aus der des Nutzers. Eine wichtige Grundlage hierfür ist das Bewertungsmodell von Ortony et al. (1988), auch bekannt als „OCC-Modell“" (nach den Namen der drei Autoren). Dieses Modell ordnet über 20 alltägliche Gefühlszustände systematisch deren Auslösern zu, wodurch es gut mit Programmieransätzen zur Handlungsplanung vereinbar ist.

Um selbst Emotionen auszudrücken, besitzen manche Roboter zusätzliche Motoren zur Bewegung von Mundwinkeln, Augenbrauen, Augenlidern oder auch Ohren unter einer Silikonhaut oder künstlichem Fell (siehe Abb. 4.2). Andere Roboter, zum Beispiel Buddy ${ }^{6}$ oder Jibo ${ }^{7}$, verzichten auf ein mechanisches Gesicht und zeigen stattdessen stilisierte Animationen auf einem Bildschirm. Ein solcher Roboter sieht zwar deutlich technischer aus, ist aber dafür weniger anfällig für Verschleiß und eröffnet zudem Möglichkeiten zur Personalisierung, da er jedes beliebige Bild anzeigen kann.

Analog zum Gesichtsausdruck ist auch die menschliche Körpersprache auf Roboter übertragbar, welche entsprechende Gelenke besitzen. Beispielsweise können sie traurig

\footnotetext{
${ }^{6}$ http://www.bluefrogrobotics.com/en/buddy/.

${ }^{7}$ https://www.jibo.com.
} 

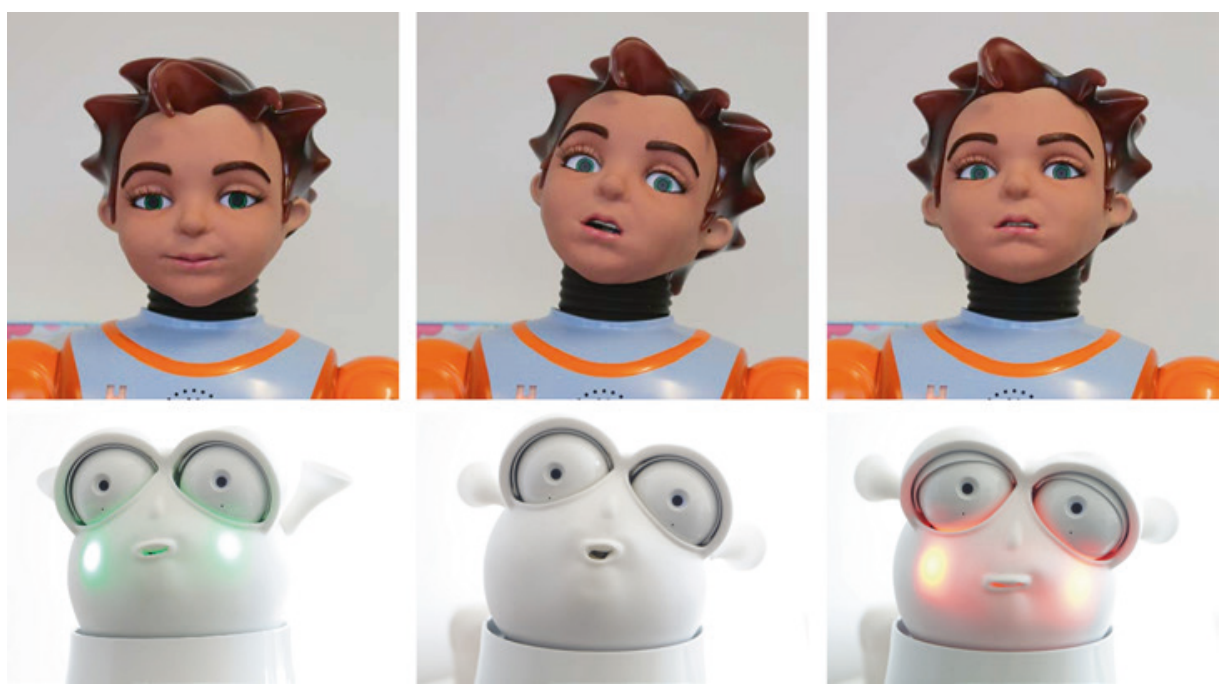

Abb. 4.2 Emotionale Mimik bei Hanson RoboKind R50 (oben) und Robopec Reeti (unten)

den Kopf hängen lassen oder aufgeregt mit den Armen gestikulieren. Allerdings ist bei diesen Animationen zusätzlich darauf zu achten, dass die Roboter nicht umfallen oder mit Personen und Gegenständen kollidieren können.

Weitere Möglichkeiten sind die Anpassung der Sprachausgabe und das Abspielen emotional behafteter Geräusche, etwa Weinen oder einer fröhlichen Fanfare, sowie der Einsatz von Farbsignalen, welche rein symbolisch sein oder physiologische Reaktionen wie Erröten und Erblassen nachahmen können. Als Beispiel sei auf eine Perzeptionsstudie von Häring et al. (2011) verwiesen, die untersucht, inwiefern sich Emotionen durch Augenfarbe und Geräusche, aber auch durch Körperposen und -bewegungen der humanoiden Roboter der Firma Aldebaran Robotics wiedergeben lassen.

\subsubsection{Sozial situiertes Lernen}

Die Anpassung des Roboters an den individuellen Nutzer spielt für Pflegeanwendungen eine bedeutende Rolle. Hierbei gilt es einerseits, die individuellen, zum aktuellen Zeitpunkt bzw. zu der aktuellen Situation vorliegenden Präferenzen des Menschen zu berücksichtigen, und andererseits den Roboter auch erlernen zu lassen, durch welches Verhalten der Nutzer langfristig engagiert werden kann. Dabei sollte auf einige Faktoren Rücksicht genommen werden, wie etwa die Persönlichkeit, Stimmung und Präferenzen des Nutzers, aber auch mögliche körperliche Einschränkungen.

Um erlernen zu können, welches Roboterverhalten in welcher Situation das beste ist, kommen oftmals Algorithmen des bestärkenden Lernens zum Einsatz. Dabei versucht der Roboter autonom unterschiedliche Verhaltensweisen und erhält dafür jeweils 
eine Belohnung oder Bestrafung. Beispielsweise benutzen Tapus et al. (2008) im Kontext eines Therapieroboters für Schlaganfallpatienten bestärkendes Lernen, um das Verhalten des Roboters entsprechend der Persönlichkeit und Performanz des Nutzers an sein Profil anzupassen. Dabei werden Introversion und Extraversion in der Sprache manipuliert sowie die Geschwindigkeit des Roboters und der Abstand zum Nutzer, wobei die Belohnung von der Anzahl der vom Nutzer ausgeführten Übungen abhängt.

Der Belohnung bzw. Bestrafung kommt hierbei eine Schlüsselrolle zu. Dieser positive oder negative Zahlenwert ist die einzige Information, anhand derer der Roboter erkennen kann, ob sein gezeigtes Verhalten in der entsprechenden Situation zielführend ist oder nicht. Eine einfache, aber gleichzeitig auch sehr aufdringliche und unelegante Möglichkeit stellt zu diesem Zweck die explizite Befragung des Nutzers dar. Um dies zu umgehen, können einerseits aufgabenspezifische Informationen - wie im Falle von Tapus et al. (2008) die Anzahl der absolvierten Rehabilitationsübungen - herangezogen werden.

Andererseits sind aber auch menschliche soziale Signale eine reiche Informationsquelle, um Wissen über den aktuellen Zustand der Person einfließen zu lassen und/oder die Belohnung abhängig von sozialen Hinweisreizen des Menschen zu gestalten, etwa menschliches Engagement (Ritsche 2017), taktiles (Barraquand und Crowley 2008) oder prosodisches (Kim und Scassellati 2007) Feedback, Blickverhalten (Mitsunaga et al. 2008), Lächeln (Leite et al. 2011; Gordon et al. 2016; Knight 2011) oder Lachen (Hayashi et al. 2008; Knight 2011; Katevas et al. 2015; Weber et al. 2018). Ein wichtiger Vorteil sozialer Signale ist hierbei der Fakt, dass diese unterbewusst und über die gesamte Zeit einer Interaktion auftreten, ohne dass der Mensch explizit befragt werden muss.

Abb. 4.3 veranschaulicht diesen Ansatz: Während der Interaktion zwischen Mensch und Roboter sendet der Nutzer permanent soziale Signale aus, die sich beispielsweise in Form von Gestik, Mimik oder Blickverhalten äußern. Mithilfe geeigneter Sensoren (Mikrofone, Webcams, Microsoft Kinect, Gaze Tracker etc.) können diese in Form von Audio und Video wahrgenommen und mithilfe geeigneter Software ausgewertet werden. Die Signalverarbeitung stellt hierbei eine erste Hürde dar, schließlich unterliegen alle Sensoren physikalischen Beschränkungen. Die daraus extrahierte Information kann

Abb. 4.3 Bestärkendes Lernen auf Basis sozialer Signale

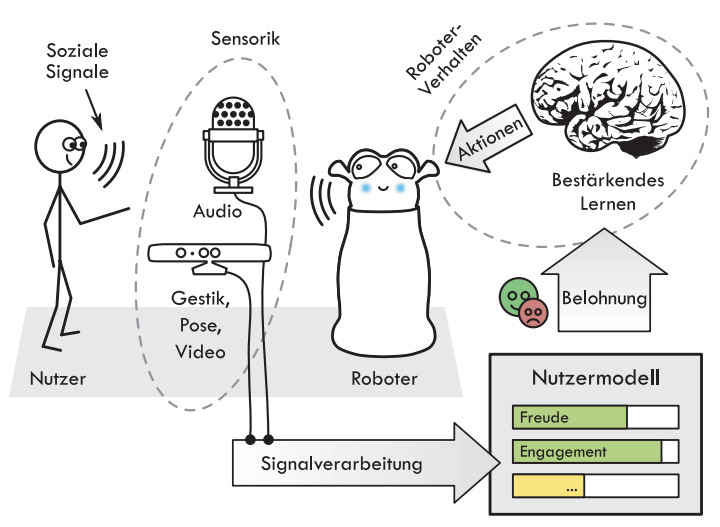


fehlerhaft sein, auch die Interpretation der Rohsignale bringt nochmals Rauschen mit sich. Auf Basis der durch die Signalverarbeitung angenäherten Informationen, beispielsweise des vom Menschen gezeigten Engagements oder seines Lächelns, kann schließlich ein Nutzermodell erstellt werden. Dieses dient dann als Eingabe für das bestärkende Lernen, das das Verhalten des Roboters steuert, der im Verlauf der Zeit erlernen kann, in welcher Situation auf Basis der Nutzerreaktion welches Verhalten den Nutzerpräferenzen am meisten entspricht.

\subsection{Anwendungen}

\subsubsection{Physiotherapie}

Ein Einsatzgebiet für Roboter ist die körperliche Rehabilitation. Beispielsweise gibt es Exoskelette, die Patienten mit eingeschränkter Beweglichkeit aktiv bei der Ausführung bestimmter Übungen unterstützen und so das Training erleichtern. Diese Roboter verfügen jedoch über keine sozialen Verhaltensweisen. Anders sieht es bei sozial assistiven Robotersystemen aus, deren primäres Ziel darin besteht, den Nutzer bei der Durchführung solcher Übungen zu motivieren.

Im ROREAS-Projekt (Gross et al. 2017) wurde ein mobiler Roboter entworfen, der Schlaganfallpatienten einer Pflegeeinrichtung beim selbstständigen Training mit ihren Gehhilfen begleitet. Zu Beginn der Trainingseinheit holt er die Patienten ab, schlägt ihnen mögliche Routen vor und fährt ihnen anschließend hinterher, um sie durch Verweise auf den bisherigen Trainingsverlauf zu motivieren oder auf Sitzmöglichkeiten zum Ausruhen aufmerksam zu machen. Falls ein Patient zu oft Pausen einlegen muss, rät der Roboter zum Umkehren oder informiert notfalls das Pflegepersonal. Wichtig hierbei war auch die automatische Navigation inmitten großer Menschenmengen, wobei der Roboter nicht nur die begleitete Person im Blick behalten, sondern auch einen angemessenen Abstand zu Unbeteiligten wahren und diesen an Engstellen den Vortritt lassen musste.

Humanoide Roboter eignen sich außerdem dazu, Patienten bei Krankengymnastik anzuleiten. Das Projekt NAOTherapist (Pulido et al. 2017) verwendet den Roboter Nao, um Kindern die Bewegungen vorzuführen, welche der Physiotherapeut für sie vorbereitet hat. Während das Kind die vorgegebene Pose nachahmt, erkennt das System mithilfe einer Kinect-Tiefenkamera, ob diese korrekt ist, und zeigt dies über die Augenfarbe des Roboters an. Bei Abweichungen macht Nao das Kind zunächst darauf aufmerksam, welcher Arm falsch ist. Reicht dies nicht, nimmt Nao kurz die gleiche Haltung ein wie das Kind, um ihm den Unterschied zwischen beiden Haltungen zu zeigen. 


\subsubsection{Gesundheitsberater}

Bei Robotern, welche als künstliche Sekretäre Termine und andere persönliche Informationen verwalten, bietet sich auch der Einsatz für medizinische Zwecke an. Beispielsweise soll der stationäre Assistent Pillo ${ }^{8}$ Familienmitglieder an Gesicht und Stimme erkennen, ihre Gesundheitsdaten zentral verwalten und sie nicht nur an die Einnahme ihrer Medikamente erinnern, sondern diese auch zur entsprechenden Zeit an die richtige Person ausgeben und bei Bedarf nachbestellen. Außerdem soll er medizinisch relevante Fragen beantworten, Videoanrufe zu Ärzten ermöglichen und Notfallkontakte über Probleme informieren.

Im Always-On-Projekt (Rich et al. 2012) berücksichtigt der Agent außerdem die aktuelle Beziehung zum Nutzer. Wenn die Vertrauensbasis durch gemeinsame Aktivitäten entsprechend aufgebaut ist, kann das System auch sensible Themen wie Ernährungstipps und sportliche Betätigung zur Sprache bringen.

Ein weiteres Einsatzfeld sind soziale Agenten, welche eine erste Anlaufstelle für Ratsuchende darstellen. Das SimCoach-Projekt (Rizzo et al. 2011) dient dazu, Militärangehörigen zu helfen, welche beispielsweise an einer posttraumatischen Belastungsstörung leiden und eine psychologische Behandlung benötigen. Im Gespräch mit dem Nutzer verschafft sich das System einen Eindruck von dessen Symptomen, weist ihn auf passende Informationsquellen und menschliche Spezialisten hin und versucht ihm durch mitfühlendes Verhalten die Scheu davor zu nehmen, professionelle Hilfe zu suchen.

Im EU-Projekt KRISTINA ${ }^{9}$ (Wanner et al. 2017) wird eine virtuelle Assistentin entwickelt, die als vertrauenswürdige Informationsquelle in Gesundheitsfragen dazu beitragen soll, sprachliche und kulturelle Barrieren im Bereich der Pflege zu überwinden. Unter anderem soll KRISTINA Migranten und unqualifizierte Angehörige von Pflegefällen bei der Kommunikation mit Pflegeeinrichtungen unterstützen.

\subsubsection{Spielgefährte}

Neben funktionalen Aspekten spielt bei sozialen Agenten auch der Unterhaltungswert eine Rolle.

Im Always-On-Projekt gehören Karten- und Brettspiele zu den Aktivitäten, welche der Agent mit allein lebenden Senioren gemeinsam unternehmen kann (Behrooz et al. 2014). Diese dienen nicht nur dem Zeitvertreib, sondern tragen gleichzeitig dazu bei, die Beziehung zwischen Agent und Nutzer zu festigen und den Weg für ernstere Themen wie dessen Gesundheit zu ebnen. Um den Effekt des gemeinsamen Spielens zu verstärken, zeigt der Agent seine Aufmerksamkeit durch menschenähnliches Blickverhalten. Zusätzlich

\footnotetext{
${ }^{8}$ https://www.pillohealth.com.

${ }^{9} \mathrm{http} / / /$ kristina-project.eu/en/.
} 
Abb. 4.4 Reeti als Partner beim Memory-Spiel

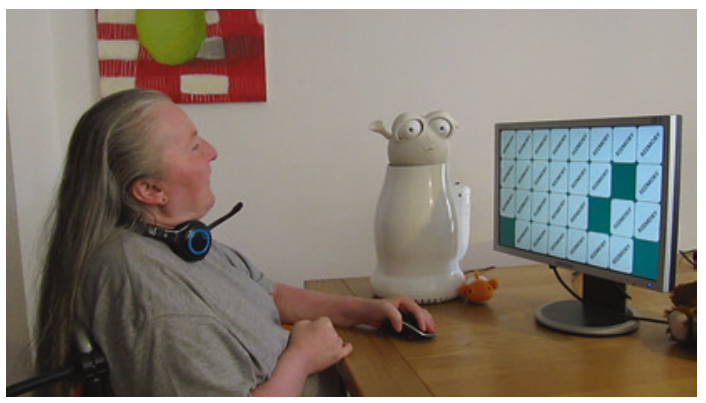

können beide Parteien sich über die aktuelle Spielsituation unterhalten, wie es auch zwischen menschlichen Spielern üblich ist.

Im Zusammenhang mit dem StMWFK-Projekt ForGenderCare ${ }^{10}$ wurde ein ähnliches System für Gesellschaftsspiele mit dem Roboter Reeti entwickelt (Mehlmann et al. 2015; McCrae und Costa 2008). Die verwendeten Spiele wie Memory und Mensch-ärgere-dich-nicht sollen dazu beitragen, die kognitiven und motorischen Fähigkeiten älterer Menschen zu erhalten (siehe Abb. 4.4). Im Mittelpunkt dieser Anwendung steht das emotionale Verhalten des Roboters, welches die langfristige Nutzung des Systems attraktiver gestalten soll. Auf Basis des Emotionsmodells ALMA (Gebhard 2007) wurden zwei verschiedene Persönlichkeiten mit unterschiedlicher Zielsetzung gestaltet. Während eine sich mit dem Nutzer freut, wenn dieser einen guten Zug macht, ist die andere auf den eigenen Sieg fixiert und reagiert daher eher feindselig. Sowohl die kurzfristigen Emotionen als auch die langfristige Stimmung des Roboters spiegeln sich in dessen Mimik und Farbsignalen wider. Die Persönlichkeit hat außerdem Einfluss darauf, welche Kommentare zum Spielgeschehen ausgewählt werden.

\subsubsection{Kuscheltier}

Obwohl die gesundheitsfördernde Wirkung von Haustieren nachgewiesen ist, können diese nicht immer in der Pflege eingesetzt werden. Dagegen sprechen beispielsweise hygienische Gründe, Allergien oder auch die Gefahr, dass kognitiv eingeschränkte Patienten das Tier vernachlässigen. In solchen Fällen können Roboter ein sinnvoller Ersatz sein.

Eines der berühmtesten Beispiele hierfür ist das künstliche Robbenbaby Paro. ${ }^{11}$ Paro kann unter anderem Berührungen und Stimmen wahrnehmen, um mit Kopf- und Flossenbewegungen sowie authentischen Tierlauten darauf $\mathrm{zu}$ reagieren. Auch verschiedene

\footnotetext{
${ }^{10} \mathrm{http} / /$ www.forgendercare.de/forschung/forschungscluster/technik-und-medien/genderaspekte-in-der-robotik-zur-altenpflege/.

${ }^{11} \mathrm{http}: / /$ www.parorobots.com.
} 
Spielzeugfirmen bieten Roboter an, welche für diese Anwendung in Betracht kommen (Heerink et al. 2013). Dazu gehören beispielsweise Hasbros FurReal Friends ${ }^{12}$ oder der Dinosaurier Pleo ${ }^{13}$.

Eine Kernfunktion dieser Roboter ist es, ihre Nutzer zu fürsorglichem Verhalten wie Streicheln, Füttern und Spielen zu ermuntern und sie dadurch aus der Reserve zu locken oder negative Gefühle wie Einsamkeit und Kummer zu lindern. Zudem können sie auch ein Werkzeug zur Selbstreflexion sein. Beispielsweise können sie auf bestimmte Reize emotional reagieren und den Nutzer dadurch motivieren, sein Verhalten zu ändern. Möglich ist auch, emotionale und physiologische Zustände zwischen Mensch und Roboter zu synchronisieren, um den Betroffenen ihren aktuellen Zustand zu veranschaulichen. Um das Potenzial dieser Anwendung zu erkunden, haben Aslan et al. (2016) ein Stofftier mit einem motorisierten Brustkorb ausgestattet. Dieser kann durch die Atembewegung eines Nutzers gesteuert werden, welche über einen Sensorgurt um dessen Brust erkannt wird. Das Stofftier soll besonders Nutzern, welche noch keine Erfahrung mit Meditation haben, den Zugang zu achtsamkeitsbasierten Techniken (auch bekannt als „Mindfulness Based Stress Reduction“ oder MBSR) erleichtern.

\subsection{Gestaltung von sozial interagierenden Robotern}

Beim Entwurf von sozial interagierenden Robotern stehen die Entwickler vor der Frage, welches Erscheinungsbild sie diesen geben sollen und welche Verhaltensweisen einzuprogrammieren sind. Zahlreiche Studien haben gezeigt, dass bereits wenige Schlüsselmerkmale genügen, damit Menschen auf Roboter oder virtuelle Charaktere ähnlich reagieren wie auf ein menschliches Gegenüber. Es ist daher zu erwarten, dass diversifizierende Merkmale von virtuellen Charakteren und Robotern ähnlich wahrgenommen werden wie diversifizierende Merkmale von Menschen.

Studien haben gezeigt, dass ältere Menschen diskrete, kleine Roboter mit menschenoder haustierähnlichem Verhalten gegenüber großen humanoiden Robotern bevorzugen (Broadbent et al. 2009; Wu et al. 2012). Darüber hinaus werden sich langsam bewegende Roboter mit weiblicher Stimme, weniger autonomem Verhalten und seriösem Aussehen mit einer höheren Wahrscheinlichkeit akzeptiert (Broadbent et al. 2009). Auch reagieren ältere Personen nachweislich positiv auf soziale Roboter, die ihr soziales Verhalten der Ernsthaftigkeit der aktuellen Aufgabe oder Situation anpassen (Goetz et al. 2003).

Roboter sollten nicht bedrohlich, sondern gutherzig (Broadbent et al. 2009; Frennert et al. 2012) in Erscheinung treten. Je realistischer das Gesicht, desto glaubwürdiger und geselliger wird der Roboter wahrgenommen (Spiekman et al. 2011). Das Erscheinungsbild allein genügt jedoch nicht. Sieht der Roboter sehr menschenähnlich aus, kann aber

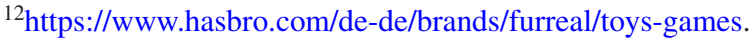

${ }^{13} \mathrm{http}: / / \mathrm{www} \cdot$ pleoworld.com/pleo_rb/eng/lifeform.php.
} 
nicht die damit einhergehenden, entsprechend hohen Erwartungen an sein Verhalten erfüllen, führt dies tendenziell zu Enttäuschung und Misstrauen (Walters et al. 2008).

Studien von Kuchenbrandt et al. (2014) und Häring et al. (2014) deuten an, dass bereits wenige Schlüsselreize wie z. B. eine männliche versus eine weibliche Computerstimme oder ein deutscher versus persischer Vorname genügen, um Roboter einer sozialen Gruppe zuzuweisen. Mit einer solchen Zuordnung werden meist unbewusst bestimmte Stereotypen aktiviert, was sich wiederum auf das Interaktionsverhalten mit dem Roboter auswirken kann. So zeigten Häring et al. (2014), dass Versuchspersonen bevorzugt mit Robotern kooperieren, die der eigenen sozialen Gruppe angehören.

Ein wesentlicher Akzeptanztreiber für die Roboter ist deren Fähigkeit, mit Menschen auf sozial angemessene Art und Weise zu interagieren. Dies beinhaltet u. a. auch das Befolgen von Höflichkeitsregeln. Eine Herausforderung bei der Interaktion ist, dass durch das Aussprechen von Empfehlungen indirekt auf vorhandene Einschränkungen oder Versäumnisse der Nutzerinnen und Nutzer hingewiesen wird. Damit besteht die Gefahr, dass sich die Menschen bevormundet oder peinlich berührt fühlen könnten. In solchen Fällen können bereits Nuancen in der Formulierung einen entscheidenden Einfluss auf die Wahrnehmung des Roboters und die Annahme der Empfehlungen haben. Daher ist es wichtig, bei der Formulierung der Empfehlungen die richtige Balance zu finden: Roboter sollten Menschen zwar auf überzeugende Weise auf mögliche Aktivitäten hinweisen, die deren Wohlbefinden steigern können, aber ohne bevormundend wahrgenommen zu werden. Intelligent ausgewählte Höflichkeitsstrategien könnten hierbei hilfreich sein und die Akzeptanz des Roboters durch die Menschen fördern (Hammer et al. 2016).

\subsection{Wirkung von sozialen Robotern}

Eine Reihe von Studien weist auf die positiven Effekte von sozial interagierenden Robotern hin. So können Roboter bei älteren Menschen das Gefühl der Einsamkeit lindern, soziale Interaktionen mit anderen Menschen stimulieren und damit insgesamt zu einer Steigerung der Lebensqualität beitragen.

Einsamkeit ist ein häufiges Phänomen bei älteren Menschen, das sich negativ auf die mentale Gesundheit auswirken kann (Losada et al. 2012). Häufig wird der Kritikpunkt vorgebracht, dass Roboter keine echte Zuwendung ersetzen können. Cohen-Mansfield et al. (2012) haben in einer Studie festgestellt, dass Roboter zwar zur Stimmungsaufhellung von Demenzpatienten beitragen können, aber nicht die Wirkung von zwischenmenschlichen Kontakten erzielen. Nichtsdestotrotz gibt es Evidenz für den therapeutischen Nutzen von simulierten sozialen Stimuli. So können Roboter Demenzpatienten dazu anregen, mit ihrem sozialen Umfeld $\mathrm{zu}$ interagieren. Untersuchungen zeigen, dass soziale Roboter selbst häufig zum Thema für Gespräche mit Pflegern oder Verwandten werden und somit eine Art Vermittlerrolle einnehmen. Denkbar ist auch, dass Enkelkinder oder andere Verwandte häufiger zu Besuch kommen, da sie neugierig auf den Roboter sind (Sharkey und Sharkey 2012). 
Als Beispiel sei auf eine Studie von Kramer et al. (2009) verwiesen, die das Potenzial von AIBO, einem von der Firma Sony entwickelten Roboterhund, im Bereich der Pflege explorierten. Der von ihnen eingesetzte AIBO-Roboter war in der Lage, sich umzuschauen, tanzähnliche Bewegungen auszuführen und mit den Vorderbeinen zu winken. Er spielte außerdem Musik ab und konnte LEDs am Kopf und Rücken zum Leuchten bringen. Es stellte sich heraus, dass sich der AIBO-Roboter positiv auf Interaktionen von Demenzpatienten mit ihrem Umfeld auswirkte.

Studien, die Aufschluss zum Langzeitnutzen von sozial interagierenden Robotern liefern könnten, sind immer noch eine Seltenheit, können aber wertvolle Hinweise zu einem dauerhaften therapeutischen Nutzen bieten. Chang et al. (2013) zeichneten über acht Wochen hinweg die Interaktionen von Demenzpatienten mit der Roboterrobbe Paro auf. Während dieser Zeit nahm der physische Kontakt (z. B. beim Streicheln oder Umarmen) zwischen den Demenzpatienten und der Roboterrobbe kontinuierlich zu, was als Indiz für den Aufbau einer emotionalen Bindung zwischen Demenzpatienten und Robotern angesehen werden kann.

Studien zur positiven Wirkung von Robotern im Bereich der Pflege können jedoch nicht über die offensichtlichen Grenzen von künstlichen Kreaturen hinwegtäuschen. Trotz technischer Fortschritte können Roboter das spontane Verhalten von Lebewesen noch nicht auf glaubhafte Art und Weise replizieren. Ein höherer Grad an Realismus im Aussehen führt dabei nicht unbedingt zu höherer Akzeptanz. Vielmehr wird bei einer allzu großen, aber dennoch nicht perfekten Ähnlichkeit zu lebenden Kreaturen ein Bereich beschritten, in dem eine künstliche Kreatur eher Unbehagen erzeugt. Dieser Bereich wird auch als „Unheimliches Tal“ (engl. ,uncanny valley“) bezeichnet (Mori et al. 2012).

Trotz aller positiven Argumente für den Einsatz sozialer Roboter bei älteren Menschen könnte argumentiert werden, dass weiter verbreitete Geräte wie Smartphones oder Tablet-Computer günstiger und durch den gewohnten Umgang mit ihnen auch einfacher zu handhaben wären. So könnten beispielsweise Handlungsempfehlungen oder Erinnerungen einfach auf dem Display angezeigt werden. Dem ist entgegenzusetzen, dass vor allem kleine Displays und Touch-Interaktion für ältere Leute oft problematisch sind (Angelini et al. 2013). In einem direkten Vergleich zwischen der Wirkung eines sozialen Roboters im Gegensatz zu einem Tablet-Computer, welche dieselben Empfehlungen präsentierten, wie regelmäßiges Trinken oder Lüften, untersuchten Hammer et al. (2017) die Handhabung beider Geräte bei älteren Menschen. Sie konnten unter anderem zeigen, dass Teilnehmer und Teilnehmerinnen der Studie die Interaktion mit dem sozialen Roboter als weniger komplex und leichter zu erlernen einschätzten als die Interaktion mit dem Tablet-Computer.

Soll ein sozialer Roboter als Unterstützung im häuslichen Umfeld eingesetzt werden, bleibt weiterhin die Frage offen, wie dieser auftreten soll, um von älteren Nutzern akzeptiert zu werden. Dabei können vielzählige Faktoren wie die Rolle des Roboters oder dessen Einhalten von sozialen Normen von Bedeutung sein. Bartl et al. (2016) ließen einen sozialen Roboter ältere Leute an Termine erinnern, entweder in der Rolle eines Gefährten oder in der Rolle eines Assistenten (Sekretär). Dabei wurden rollentypische 
Verhaltensweisen wie professionelle oder freundschaftliche Sprache oder die Häufigkeit des gezeigten Lächelns variiert. Interessanterweise wurde der Roboter in der Rolle als Gefährte nicht nur eher akzeptiert und als freundlicher empfunden, sondern auch als kompetenter bewertet. Diese Ergebnisse legen nahe, dass bei der Umsetzung von Assistenzsystemen für ältere Menschen besonders auf die Integration von sozialen Verhaltensaspekten geachtet werden sollte.

\subsection{Konklusion}

Sozial-interaktive Roboter unterscheiden sich von reinen Pflegerobotern durch ihre Fähigkeit, das Kommunikationsverhalten von Menschen oder Tieren nachzuahmen. Dadurch ist nicht nur die Handhabung dieser Geräte mit einem geringeren Lernaufwand verbunden, sondern es steigt auch die Bereitschaft von pflegebedürftigen Personen, diese Hilfsmittel zu nutzen.

Noch sind viele Herausforderungen zu bewältigen, um Roboter mit all den sozialen Kompetenzen auszustatten, welche sie für den Einsatz in diesem zutiefst persönlichen Aufgabenfeld benötigen. Die Wahrnehmung und Interpretation menschlicher Signale insbesondere solcher, deren Bedeutung von der jeweiligen Situation abhängt - wird zwar immer weiter verbessert, muss aber noch den Schritt vom Labor in die Realität schaffen, wo sie mit der unvorhersehbaren Vielfalt menschlicher Ausdrucksweisen, Familien- und Wohnsituationen konfrontiert wird. Auch in puncto Datenschutz und Privatsphäre sind noch wichtige Fragen zu klären. Pflege ist immer mit einem gewissen Grad an Abhängigkeit verbunden, weswegen es zwingend erforderlich ist, dass die Betroffenen ihren künstlichen Assistenten vertrauen können. Beim Erscheinungsbild und Verhalten der Roboter muss noch die richtige Balance zwischen Realismus und Stilisierung gefunden werden, welcher von unzähligen Faktoren wie dem konkreten Einsatzzweck, persönlichen Vorlieben des Nutzers und den tatsächlichen Fähigkeiten des Roboters abhängt.

Die hier genannten Studien zeigen, dass soziale Roboter bereits auf dem heutigen Stand der Technik ein wertvolles Werkzeug sein können, um die Lebensqualität pflegebedürftiger Personen zu steigern und Brücken zwischen ihnen und ihren Mitmenschen zu bauen. Ähnlich wie bei Heimcomputern, Mobiltelefonen und Speichermedien ist auch bei Robotern damit zu rechnen, dass die benötigte Technologie zunehmend leistungsfähiger, kleiner und kostengünstiger werden wird. Während weltweit Forschungsprojekte gefördert werden, um die beschriebenen Herausforderungen zu lösen, steigt auch der Anteil der Bevölkerung, welcher mit derartigen Assistenztechnologien und Zukunftsvisionen aufwächst. Entsprechend werden sich die Fähigkeiten sozialer Roboter und die Bereitschaft der Menschen, diese zu nutzen, einander weiter annähern, und die Vision vom hilfreichen Robotergefährten wird sowohl in professionellen Pflegeeinrichtungen als auch in den eigenen vier Wänden langsam, aber sicher zur Realität werden.

Danksagung Die hier beschriebene Arbeit wurde teilweise unterstützt durch das STMWFK im Rahmen des Forschungsverbundes ForGenderCare. 


\section{Literatur}

André, E. (2014). Lässt sich Empathie simulieren? Ansätze zur Erkennung und Generierung empathischer Reaktionen anhand von Computermodellen. Nova Acta Leopoldina NF, 120(405), 81-105.

Angelini, L., Caon, M., Carrino, S., Bergeron, L., Nyffeler, N., Jean-Mairet, M., \& Mugellini, E. (2013). Designing a desirable smart bracelet for older adults. Proceedings of the 2013 ACM conference on pervasive and ubiquitous computing adjunct publication (S. 425-434). ACM.

Aslan, I., Burkhardt, H., Kraus, J., \& André, E. (2016). Hold my heart and breathe with me: Tangible somaesthetic designs. Proceedings of the 9th Nordic conference on human-computer interaction (S. 92). ACM.

Aylett, MP., Vinciarelli, A., \& Wester, M. (2017). Speech synthesis for the generation of artificial personality. IEEE transactions on affective computing, Early Access Articles. IEEE.

Barraquand, R., \& Crowley, J .L. (2008). Learning polite behavior with situation models. Proceedings of the 3rd ACM/IEEE international conference on human robot interaction (S. 209-216). ACM.

Bartl, A., Bosch, S., Brandt, M., Dittrich, M., \& Lugrin, B. (2016). The Influence of a social robot's persona on how it is perceived and accepted by elderly users. International Conference on Social Robotics (S. 681-691). Cham: Springer.

Behrooz, M., Rich, C., \& Sidner, C. (2014). On the sociability of a game-playing agent: A software framework and empirical study. Intelligent Virtual Agents, Lecture Notes in Computer Science, 8637, (S. 40-53). Cham: Springer.

Bickmore, T., \& Cassell, J. (1999). Small talk and conversational storytelling in embodied conversational interface agents. AAAI Fall Symposium on Narrative Intelligence (S. 87-92).

Bickmore, T., \& Cassell, J. (2001). Relational agents: A model and implementation of building user trust. Proceedings of the SIGCHI conference on human factors in computing systems (S. 396-403). ACM.

Breazeal CL (2004). Designing sociable robots. MIT Press.

Broadbent, E., Stafford, R., \& MacDonald, B. (2009). Acceptance of healthcare robots for the older population: Review and future directions. International Journal of Social Robotics, 1(4), 319-330.

Chang, W. L., Sabanovic, S. \& Huber, L. (2013, October). Situated analysis of interactions between cognitively impaired older adults and the therapeutic robot PARO. In Proceedings of 5th International Conference of Social Robotics (ICSR 2013), Bristol, UK, 27-29, 2013 (S. 371-380).

Chung, H., Iorga, M., Voas, J., \& Lee, S. (2017). “Alexa, Can I Trust You?” Computer, 50(9), 100-104. IEEE.

Cohen-Mansfield, J., Marx, MS., Freedman, L. S., Murad, H., Thein, K., \& Dakheel-Ali, M. (2012). What affects pleasure in persons with advanced stage dementia? Journal of Psychiatric Research, 46(3), 402-406.

Ekman, P., Friesen, W. V., \& Hager, J. C. (2002). Facial action coding system: The manual on CD ROM. Salt Lake City: A human face.

Forsa. Politik- und Sozialforschung GmbH. (2016). Service-Robotik: Mensch-Technik-Interaktion im Alltag: Ergebnisse einer repräsentativen Befragung. Berlin, Deutschland. https://www.bmbf. de/files/BMBF_forsa_Robotik_FINAL2016.pdf.

Frennert, S., Östlund, B., \& Eftring, H. (2012). Would granny let an assistive robot into her home? International Conference on Social Robotics (S. 128-137). Berlin: Springer.

Gebhard, P. (2007). Emotionalisierung interaktiver Virtueller Charaktere. Ein mehrschichtiges Computermodell zur Erzeugung und Simulation von Gefühlen in Echtzeit. Doktorarbeit, Universität des Saarlandes. 
Goetz, J., Kiesler, S., \& Powers, A. (2003). Matching robot appearance and behavior to tasks to improve human-robot cooperation. In Proceedings of the 12th IEEE International Workshop on Robot and Human Interactive Communication (ROMAN 2003) (S. 55-60).

Gordon, G., Spaulding, S., Westlund, J. K., Lee. J. J., Plummer, L., Martinez, M., Das, M., \& Breazeal, C. (2016) Affective personalization of a social robot tutor for children's second language skills. Proceedings of the thirtieth AAAI conference on artificial intelligence (S. 3951-3957). AAAI Press.

Gross, H. M., Scheidig, A., Debes, K., Einhorn, E., Eisenbach, M., Mueller, S., Schmiedel, T., Trinh, T. Q., Weinrich, C., Wengefeld, T., Bley, A., \& Martin, C. (2017). ROREAS: Robot coach for walking and orientation training in clinical post-stroke rehabilitation - Prototype implementation and evaluation in field trials. Autonomous Robots, 41(3), 679-698.

Hammer, S., Lugrin, B., Bogomolov, S., Janowski, K., \& André, E. (2016). Investigating politeness strategies and their persuasiveness for a robotic elderly assistant. 11th International Conference on Persuasive Technology (S. 315-326). Cham: Springer.

Hammer, S., Kirchner, K., André, E., \& Lugrin, B. (2017). Touch or talk? - Comparing social robots and tablet PCs for an elderly assistant recommender system. Proceedings of the Companion of the 2017 ACM/IEEE International Conference on Human-Robot Interaction (S. 129-130). ACM.

Häring, M., Bee, N., \& André, E. (2011). Creation and evaluation of emotion expression with body movement, sound and eye color for humanoid robots. 2011 IEEE RO-MAN (S. 204-209).

Häring, M., Kuchenbrandt, D., \& André, E. (2014). Would you like to play with me? How robots' group membership and task features influence human-robot interaction. In proceedings of the 2014 ACM/IEEE International Conference on Human-Robot Interaction (S. 9-16).

Hayashi, K., Kanda, T., Miyashita, T., Ishiguro, H., \& Hagita, N. (2008). Robot manzai: Robot conversation as a passive - Social medium. International Journal of Humanoid Robotics, 5(1), 67-86.

Heerink, M., Albo-Canals, J., Valenti-Soler, M., Martinez-Martin, P., Zondag, J., Smits, C., \& Anisuzzaman, S. (2013). Exploring requirements and alternative pet robots for robot assisted therapy with older adults with dementia. International Conference on Social Robotics (S. 104-115). Cham: Springer.

Huijnen, C., Badii, A., Heuvel, H. van den, Caleb-Solly, P., \& Thiemert, D. (2011). "Maybe it becomes a buddy, but do not call it a robot" - Seamless cooperation between companion robotics and smart homes. International Joint Conference on Ambient Intelligence (S. 324-329). Berlin: Springer.

Katevas, K., Healey, P. G., \& Harris, M. T. (2015). Robot comedy lab: Experimenting with the social dynamics of live performance. Frontiers in Psychology, 6, 1253.

Kim, E. S., \& Scassellati, B. (2007). Learning to refine behavior using prosodic feedback. In Proceedings 2007 IEEE 6th International Conference on Development and Learning (ICDL 2007) (S. 205-210). IEEE.

King, C. H., Chen, T. L., Jain, A., \& Kemp, C. C. (2010). Towards an assistive robot that autonomously performs bed baths for patient hygiene. The 2010 IEEE/RSJ International Conference on Intelligent Robots and Systems (S. 319-324). IEEE.

Knight, H. (2011). Eight lessons learned about non-verbal interactions through robot theater. International Conference on Social Robotics (S. 42-51). Berlin: Springer.

Kramer, S. C., Friedmann, E., \& Bernstein, P. L. (2009). Comparison of the effect of human interaction, animal-assisted therapy, and AIBO-assisted therapy on long-term care residents with dementia. Anthrozoos: A Multidisciplinary Journal of the Interactions of People \& Animals, 22(1), 43-57. 
Kuchenbrandt, D., Häring, M., Eichberg, J., Eyssel, F., \& André, E. (2014). Keep an eye on the task! how gender typicality of tasks influence human-robot interactions. International Journal of Social Robotics, 6(3), 417-427. Springer.

Leite, I., Pereira, A., Castellano, G., Mascarenhas, S., Martinho, C., \& Paiva, A. (2011). Modelling empathy in social robotic companions. International Conference on User Modeling, Adaptation, and Personalization (S. 135-147). Berlin: Springer.

Lim, M. Y., Aylett, R., Ho, W. C., Enz, S., \& Vargas, P. (2009). A socially-aware memory for companion agents. International Workshop on Intelligent Virtual Agents (S. 20-26). Berlin: Springer.

Losada, A., Márquez-González, M., García-Ortiz, L., Gómez-Marcos, M. A., Fernández-Fernández, V., \& Rodríguez-Sánchez, E. (2012). Loneliness and mental health in a representative sample of community-dwelling Spanish older adults. The Journal of Psychology, 146(3), 277-292.

Mairesse, F., \& Walker, M. A. (2011). Controlling user perceptions of linguistic style: Trainable generation of personality traits. Computational Linguistics, 37(3), 455-488.

McCrae, R. R., \& Costa Jr, P. T. (2008). The five-factor theory of personality. Handbook of personality: Theory and research, 3, 159-181.

Mehlmann, G., Häring, M., Janowski, K., Baur, T., Gebhard, P., \& André, E. (2014). Exploring a model of gaze for grounding in multimodal HRI. Proceedings of the 16th International Conference on Multimodal Interaction (S. 247-254).

Mehlmann, G., Janowski, K., \& André, E. (2015). Modeling grounding for interactive social companions. KI - Künstliche Intelligenz, 30(1), 45-52.

Mitsunaga, N., Smith, C., Kanda, T., Ishiguro, H., \& Hagita, N. (2008). Adapting robot behavior for human-robot interaction. IEEE Transactions on Robotics, 24(4), 911-916.

Mizak, A., Park, M., Park, D., \& Olson, K. (2017). Amazon "Alexa" pilot analysis report. Front Porch Center for Innovation and Wellbeing.

Mori, M., MacDorman, K. F., \& Kageki, N. (2012). The uncanny valley. IEEE Robotics \& Automation Magazine, 19(2), 98-100. IEEE.

Ortony, A., Clore, G. L., \& Collins, A. (1988). The cognitive structure of emotions. Cambridge University Press.

Pino, M., Boulay, M., Jouen, F., \& Rigaud, A. A. (2015). "Are we ready for robots that care for us?" Attitudes and opinions of older adults toward socially assistive robots. Frontiers in Aging Neuroscience, 7, 141.

Pulido, J. C., González, J. C., Suárez-Mejías, C., Bandera, A., Bustos, P., \& Fernández, F. (2017). Evaluating the child-robot interaction of the NAOTherapist platform in pediatric rehabilitation. International Journal of Social Robotics, 9(3), 343-358.

Reeves, B., \& Nass, C. (1998). The media equation: How people treat computers, television, and new media like real people and places. Cambridge: Cambridge University Press.

Reis, A., Paulino, D., Paredes, H., \& Barroso, J. (2017). Using intelligent personal assistants to strengthen the elderlies' social bonds - A preliminary evaluation of amazon Alexa, Google Assistant, Microsoft Cortana, and Apple Siri. International Conference on Universal Access in Human-Computer Interaction (S. 593-602). Cham: Springer.

Rich, C., Sidner, C. L., Nooraei, B., \& Coon, W. (2012). Operating in a hierarchy of time scales for an always-on relational agent. Workshop on Real-Time Conversations with Virtual Agents, Santa Cruz, CA.

Ritschel, H., Baur, T., \& André, E. (2017). Adapting a robot's linguistic style based on socially-aware reinforcement learning. In 26th IEEE International Symposium on Robot and Human Interactive Communication (RO-MAN 2017) (S. 378-384).

Rizzo, A. A., Lange, B., Buckwalter, J. G., Forbell, E., Kim, J., Sagae, K., Williams, J., Rothbaum, B. O., Difede, J., Reger, G., Parsons, T., \& Kenny, P. (2011). An intelligent virtual human sys- 
tem for providing healthcare information and support. Studies in health technology and informatics, 163, 503-509.

Sabelli, A. M., Kanda, T., \& Hagita, N. (2011). A conversational robot in an elderly care center: An ethnographic study. Proceedings of the 6th International Conference on Human-Robot Interaction (S. 37-44). ACM.

Serban, I. V., Sankar, C., Germain, M., Zhang, S., Lin, Z., Subramanian, S., Kim, T., Pieper, M., Chandar, S., Ke, N. R., Rajeswar, S., Brebisson, A. de., Sotelo, J. M. R., Suhubdy, D., Michalski, V., Nguyen, A., Pineau, J., \& Bengio, Y. (2018). A deep reinforcement learning chatbot (Short Version). ArXiv preprint arXiv:1801.06700.

Sharkey, A., \& Sharkey, N. (2012). Granny and the robots: Ethical issues in robot care for the elderly. Ethics and Information Technology, 14(1), 27-40.

Sidner, C., Bickmore, T., Rich, C., Barry, B., Ring, L., Behrooz, M., \& Shayganfar, M. (2013). An always-on companion for isolated older adults. 14th Annual SIGdial Meeting on Discourse and Dialogue.

Spiekman, M., Haazebroek, P., \& Neerincx, M. (2011). Requirements and platforms for social agents that alarm and support elderly living alone. International Conference on Social Robotics (S. 226-235).

Srinivasa, S. S., Ferguson, D., Helfrich, C. J., Berenson, D., Collet, A., Diankov, R., Gallagher, G., Hollinger, G., Kuffner, J., \& Vande Weghe, M. (2010). HERB: A Home Exploring Robotic Butler. Autonomous Robots, 28(1), 5-20.

Tapus, A., Mataric, M. J., \& Scassellati, B. (2007). Socially assistive robotics [Grand Challenges of Robotics]. IEEE Robotics \& Automation Magazine, 14(1), 35-42.

Tapus, A., Ţăpuş, C., \& Matarić, M. J. (2008). User - Robot personality matching and assistive robot behavior adaptation for post - Stroke rehabilitation therapy. Intelligent Service Robotics, $1(2), 169-183$.

Vogt, T., \& André, E. (2005). Comparing feature sets for acted and spontaneous speech in view of automatic emotion recognition. Proceedings of the 2005 IEEE International Conference on Multimedia and Expo (ICME 2005) (S. 474-477).

Vogt, T., André, E., \& Bee, N. (2008). EmoVoice - A framework for online recognition of emotions from voice. Perception in multimodal dialogue systems (S. 188-199).

Walker, M. A., Sawyer, J., Lin, G., \& Wing, S. (2014). Does personality matter? Expressive generation for dialogue interaction. Natural interaction with robots, knowbots and smartphones (S. 285-301). New York: Springer.

Walters, M., Syrdal, D., Dautenhahn, K., Boekhorst, R. te., \& Koay, K. (2008). Avoiding the uncanny valley: Robot appearance, personality and consistency of behavior in an attentionseeking home scenario for a robot companion. Autonomous Robots, 24(2), 159-178.

Wanner, L., André, E., Blat, J., Dasiopoulou, S., Farrùs, M., Fraga, T., Kamateri, E., Lingenfelser, F., Llorach, G., Martínez, O., Meditskos, G., Mille, S., Minker, W., Pragst, L., Schiller, D., Stam, A., Stellingwerff, L., Sukno, F., Vieru, B., \& Vrochidis, S. (2017). Kristina: A knowledgebased virtual conversation agent. International conference on practical applications of agents and multi-agent systems (S. 284-295).

Weber, K., Ritschel, H., Lingenfelser, F., \& André, E. (2018). How to shape the humor of a robot? Behavioral adaptation based on continuous social cues. AAMAS 2018 (im Druck).

Wu, Y. H., Fassert, C., \& Rigaud. A. S. (2012). Designing robots for the elderly: Appearance issue and beyond. Archives of Gerontology and Geriatrics, 54(1), 121-126. 
M.Sc. Kathrin Janowski erhielt an der Universität Augsburg den Bachelor und Master im Fach „Informatik und Multimedia“. Seit 2013 arbeitet sie am Lehrstuhl für Multimodale MenschTechnik-Interaktion und hat als wissenschaftliche Mitarbeiterin dort die Hauptverantwortung für die sozialen Roboter übernommen. Schwerpunkt ihrer Forschung ist die Generierung lebensähnlichen Verhaltens für computergesteuerte Charaktere, beispielsweise durch angemessenes Blickverhalten und den Ausdruck simulierter Emotionen. Zurzeit erforscht sie in ihrer Doktorarbeit, wie Rederechtskonflikte zwischen Mensch und Maschine in Abhängigkeit von der Gesprächssituation behandelt werden können. Bei ihrer Lehrtätigkeit steht die Entwicklung dialogbasierter Nutzerschnittstellen im Vordergrund, und seit 2014 betreut sie im Rahmen des Girls'Day Workshops, welche zukünftige Studierende an die Mensch-Roboter-Interaktion heranführen.

M.Sc. Hannes Ritschel studierte Informatik an der Universität Augsburg. Bereits in jungen Jahren kam er mit Robotern in Berührung und setzte sich im Laufe des Studiums unter anderem mit humanoiden Robotern auseinander. Seit dem Master-Abschluss 2015 arbeitet er als wissenschaftlicher Mitarbeiter am Lehrstuhl für Multimodale Mensch-Technik-Interaktion an der Universität Augsburg und untersucht in seiner Doktorarbeit bestärkendes Lernen für soziale Roboter. Schwerpunkt dabei ist das Einbeziehen menschlicher sozialer Signale in den Lernprozess, um die Maschine an die Präferenzen des Nutzers anzupassen. Neben der technischen Implementierung autonom lernender Mensch-Roboter-Schnittstellen auf Basis unterschiedlicher Roboterplattformen hält er verschiedene Lehrveranstaltungen, darunter die eigens ins Leben gerufene Vorlesung sowie ein vertiefendes Praktikum über bestärkendes Lernen. Zudem referiert Ritschel regelmäßig im Rahmen öffentlicher Veranstaltungen.

Prof. Dr. Birgit Lugrin (maiden name Birgit Endrass) is a professor for Media Informatics at University of Wuerzburg, Germany. Before, she was a faculty member at the Human Centered Multimedia Lab at Augsburg University, Germany. In her research, she focuses on the integration of diversity factors into computational models and novel interfaces such as virtual characters and humanoid robots. Her special focus lies on modelling and evaluating the (verbal and non-verbal) behaviour of virtual and robotic companions that are designed to match different user context, e.g. depending on their age or cultural background. For her doctoral thesis entitled „Cultural Diversity for Virtual Characters" Lugrin received the prestigious IFAAMAS Victor Lesser distinguished dissertation award, and the research award of Augsburg University (Wissenschaftspreis der Universität Augsburg).

Professor Dr. Elisabeth André ist Inhaberin des Lehrstuhls für Multimodale Mensch-TechnikInteraktion am Institut für Informatik der Universität Augsburg. Dort beschäftigt sie sich mit der Erforschung neuer Paradigmen für die Mensch-Roboter-Interaktion. Vorrangiges Ziel ist die Entwicklung natürlicher Interaktionsformen, die an der zwischenmenschlichen Kommunikation orientiert sind und auf Gestik, Körperhaltung, Gesichtsausdrücken und Sprache basieren. Die Forschungsarbeiten von Elisabeth André wurden durch zahlreiche Auszeichnungen gewürdigt, u. a. einen European IT Prize, einen RoboCup Scientific Award und mehrere Best Paper Awards (z. B. auf der ACM International Conference on Intelligent User Interfaces). Für ihre herausragenden Forschungsleistungen wurde sie zum Mitglied in drei wissenschaftliche Gelehrtenvereine gewählt: die Nationale Akademie der Wissenschaften Leopoldina, die Academy of Europe und AcademiaNet. Im Jahr 2013 wurde sie in die Reihe der ECCAI Fellows aufgenommen, ein Programm, mit dem Forscher gewürdigt werden, die maßgeblich das Gebiet der Künstlichen Intelligenz geprägt und zu dessen Weiterentwicklung beigetragen haben. Im Jahr 2017 erhielt sie für ihre richtungsweisenden Forschungsarbeiten auf dem Gebiet Mensch-Technik-Interaktion einen ACM SIGCHI Award und wurde als zweites Mitglied aus Deutschland überhaupt in die Computer-Human Interaction (CHI) Academy aufgenommen. 
Open Access Dieses Kapitel wird unter der Creative Commons Namensnennung 4.0 International Lizenz (http://creativecommons.org/licenses/by/4.0/deed.de) veröffentlicht, welche die Nutzung, Vervielfältigung, Bearbeitung, Verbreitung und Wiedergabe in jeglichem Medium und Format erlaubt, sofern Sie den/die ursprünglichen Autor(en) und die Quelle ordnungsgemäß nennen, einen Link zur Creative Commons Lizenz beifügen und angeben, ob Änderungen vorgenommen wurden.

Die in diesem Kapitel enthaltenen Bilder und sonstiges Drittmaterial unterliegen ebenfalls der genannten Creative Commons Lizenz, sofern sich aus der Abbildungslegende nichts anderes ergibt. Sofern das betreffende Material nicht unter der genannten Creative Commons Lizenz steht und die betreffende Handlung nicht nach gesetzlichen Vorschriften erlaubt ist, ist für die oben aufgeführten Weiterverwendungen des Materials die Einwilligung des jeweiligen Rechteinhabers einzuholen.

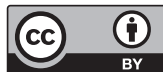

